\title{
Structural Configuration of Myelin Figures Using Fluorescence Microscopy
}

\author{
Lobat Tayebi, ${ }^{1}$ Masoud Mozafari, ${ }^{1}$ Daryoosh Vashaee, ${ }^{2}$ and Atul N. Parikh ${ }^{3}$ \\ ${ }^{1}$ Helmerich Advanced Technology Research Center, School of Material Science and Engineering, Oklahoma State University, Tulsa, \\ OK 74106, USA \\ ${ }^{2}$ Helmerich Advanced Technology Research Center, School of Electrical and Computer Engineering, Oklahoma State University, Tulsa, \\ OK 74106, USA \\ ${ }^{3}$ Department of Chemical Engineering and Material Science and Department of Biomedical Engineering, University of California, \\ Davis, CA 95616, USA
}

Correspondence should be addressed to Lobat Tayebi, lobat.tayebi@okstate.edu and Atul N. Parikh, anparikh@ucdavis.edu

Received 5 May 2012; Accepted 18 October 2012

Academic Editor: Korkut Yegin

Copyright ( 2012 Lobat Tayebi et al. This is an open access article distributed under the Creative Commons Attribution License, which permits unrestricted use, distribution, and reproduction in any medium, provided the original work is properly cited.

\begin{abstract}
Using epifluorescence microscopy, the configuration of myelin figures that are formed upon hydration of lipid stack was studied qualitatively. Little knowledge is currently available for conditions that determine the diameter of myelin figures and their degree of multilamellarity. Examining more than 300 samples, we realized that there are distinct populations of myelin figures protruding from discrete regions of lipid stack. Each population contains myelin figures with similar diameters. This indicates a direct relationship between local characteristics of parent lipid stack and the diameter of myelin figures. Evidenced by fluorescent images, we classified all the observed myelin figures into three major groups of (1) solid tubes, (2) thin tethers, and (3) hollow tubes. Solid tubes are the most common structure of myelin figures which appeared as dense shiny cylinders. Thin tethers, with long hair-shaped structure, were observed protruding from part of lipid plaque which is likely to be under tension. Hollow tubes were protruded from the parts that are unpinned from the substrate and possibly under low or no tension. The abrupt change in the configuration of myelin figures from solid tubes to hollow ones was described in a reproducible experiment where the pinned region of the parent stack became unpinned. Our observations can indicate a relation between the membrane tension of the source material and the diameter of the myelin figures.
\end{abstract}

\section{Introduction}

Concentrated mesophases of membrane-forming lipids in water deform readily producing multilamellar cylindrical tubules in the presence of external forces via a close interplay of fluidity and elasticity $[1,2]$. Similar multilamellar tubes, also known as myelin figures, are typically originating from and connected to reservoir of dense lipid plaques [3].

Such multilamellar myelin figures which consist of many different diameters [4] can be found in various types of biological organelles and cells in their normal or diseased shapes [5]. Although they can be established as transient structures too, many of them are long lived with very slow dynamics, which can be assumed as quasi-equilibrium structures such as the myelin figures in aged red blood cell [6], kidney [7], and lung $[8,9]$. A prominent example is the existence of myelin figure in pulmonary lining, an extracellular lipidprotein coat at the alveolar surface, which facilitates decrease in surface tension when lungs deflate [10].

A common property of many of these living myelin structures is that they are stable protrusions from concentrated lipid plaques in body $[3,11]$.

Other than different phospholipids which are an important source for myelin figure formation $[2,12,13]$, ionic surfactants including Aerosol OT (AOT) [14, 15] and nonionic surfactants including pentaethylene glycol lauryl ether (C12E5) [16] and triethylene glycol lauryl ether (C12E3) $[15,17]$ can also produce myelin figures. 
Beyond their biological significance, membrane tubules are also important, for instance, in the realization of artificial nanofluidic networks $[18,19]$.

Myelin figures can protrude from different sources due to different reasons especially in complex biological systems. However, the structure and configuration of all myelin figures is very similar to what is formed via simple hydration of dry lipid stack. Thus, this simple in vitro experiment is usually replaced to study the features of myelin figure production [1, 12, 14, 20-23].

Although unilamellar lipid tethers, such as those obtained by exerting local force on giant unilamellar vesicles, have been extensively studied both theoretically and experimentally [24], efforts aimed purportedly on studying the various configuration of myelin figures and how their diameters correlate with the experimental condition are sparse.

In this paper, using fluorescence microscopy, we studied the life time and different structural morphologies of myelin figures in the experiment of hydrating different kinds of dry lipid plaque. The experiments were done employing lipid stacks with various densities at different temperatures on both hydrophobic and hydrophilic substrates. We categorized the observed multilamellar myelin figures into three major groups of (1) solid tubes, (2) thin tethers, and (3) hollow tubes. These three groups were usually protruded in distinct population from separate regions of parent lipid stack. This can be an evidence to show the relation between the local characteristic of parent lipid stack and the myelin figure configuration. However, in a systematic set of experiments, we could not find a direct correlation between the diameter of myelin figures and the thickness of parent lipid stack.

\section{Material and Method}

1,2-dimyristoyl-sn-glycero-3-phosphocholine (DMPC), 1pal-mitoyl-2-oleoyl-sn-glycero-3-phosphocholine (POPC) and 1,2-dioleoyl-sn-glycero-3-phosphocholine were purchased from Avanti Polar Lipids. Texas Red DHPE and fluorescein sodium salt were purchased from Invitrogen and Sigma Aldrich, respectively.

The solutions of lipid and chloroform were prepared at different concentrations $(0.5 \mathrm{~m} / \mathrm{mL}-250 \mathrm{mg} / \mathrm{mL}) \cdot 1 \mathrm{~mol} \%$ of Texas Red DHPE was added to the solution as a sfluorescence marker.

$0.5-1 \mu \mathrm{L}$ of the lipid/chloroform solution was dropped on a substrate. The drops were air-dried for 10 minutes at room condition and were placed in vacuum for 12 hours for complete evaporation of chloroform. After this procedure, the lipid plaque (dry drop) was completely pinned to the substrate. $3-5 \mathrm{~mL}$ of DI water was added to a small Petri dish containing the dry lipid drop on a substrate. The water covers the total area of the substrate. Myelin figures start growing from the edge of the lipid drop right after hydration above the phase transition temperature $\left(T_{m}\right)$ of the lipid.

The experiments were done with various kinds of lipids including DMPC, POPC, and DOPC and were examined

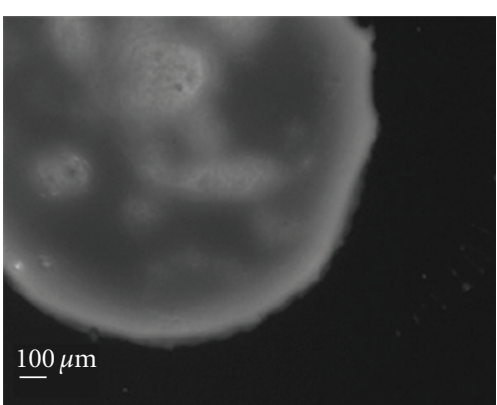

(a)

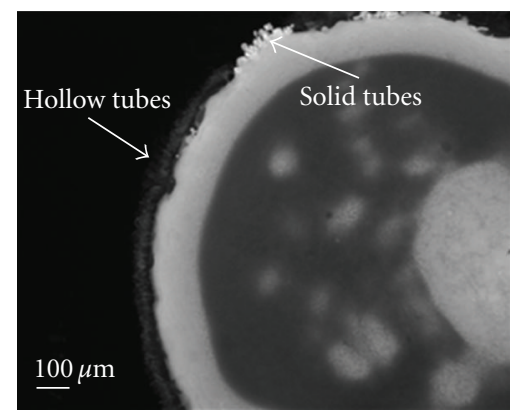

(b)

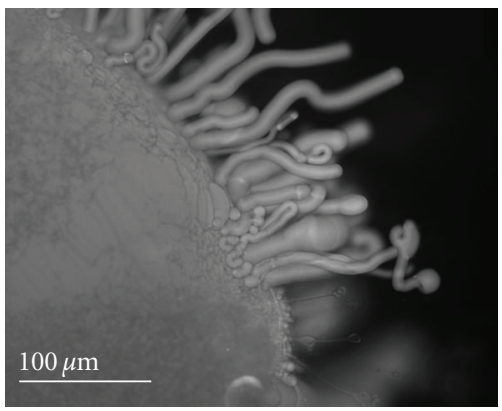

(c)

FIGURE 1: Fluorescent images of (a) dry lipid plaque (b) after myelin figure formation upon hydration. As can be seen different groups of myelin figures are protruding from distinct regions (c) higher magnification of solid tubes.

on both hydrophobic and hydrophilic substrates. Freshly piranha-etched clean glass slides were employed as a hydrophilic substrate. As hydrophobic substrates, we used silane-coated glass slides.

\section{Result and Discussions}

The dry lipid plaque and the myelin figures that are formed after hydration of the plaque were imaged using an inverted epifluorescence microscope (Nikon, Melville, NY). The typical form of a dry lipid plaque before hydration and the drop decorated by myelin figures after hydration are shown in Figures 1(a) and 1(b).

The lifetime of myelin figures was affected by the condition of the experiment. It was longer in the experiments using hydrophobic substrate than in the ones using a hydrophilic substrate. Also experiments with different initial 
concentrations of lipid/chloroform solution indicated that higher concentrations make more stable myelin figures. Both of the above conditions (quality of the substrate and the initial concentration of lipid solution) had direct influence on the configuration of dry lipid plaque. The dry drops on the hydrophobic substrate were circular in shape with welldefined dense edge. However, on the hydrophilic substrate most of the drops had a crooked shape with uneven and thin edge. Configuration of the dry drop also depended on the initial concentration of lipid/chloroform solution. The higher the concentration of the solution is, the denser and the thicker is the resulting dry lipid plaque. Since the material source of the myelin figures was the local stack of lipid they were protruding from, myelin figures were more stable when the parent lipid plaque was denser.

Also, the lifetime of myelin figures was related to the temperature. The experiments were done at different temperatures ranging from $2^{\circ} \mathrm{C}$ to $40^{\circ} \mathrm{C}$. The hydrating water was also kept at the set temperature. Myelin figures are more stable at low temperature most likely due to the slower dynamics.

Based on our experimental result, the lifetime of myelin figures can be varied from less than a minute to about 15 hours. For example, myelin figures at $2^{\circ} \mathrm{C}$ protruded from a dense lipid plaque dried from a solution of lipid/chloroform at $250 \mathrm{mg} / \mathrm{mL}$ on hydrophobic substrate could be easily stable more than 13 hours. On the contrary, hydration of dry lipid plaque from initial solution concentration of $0.5 \mathrm{mg} / \mathrm{mL}$ on hydrophilic substrate at $40^{\circ} \mathrm{C}$ could not make myelin figures with lifetime more than a minute. We concluded that the lower temperature, higher concentration (denser parent lipid plaque), and hydrophobicity of the substrate increase the stability of myelin figures.

Although there are correlations between the lifetime of myelin figures and the experimental condition, we hardly could find any relation between the diameter of the tubular structure of myelin figures and the conditions of experiments such as temperature and concentration of initial solution.

The exact reason for having myelin figures in different tubular diameters is not clearly understood yet and has been an open question for many years $[1,17]$. In a recent study [1], it was suggested that the diameter of a myelin figure is directly comparable to the thickness of the parent lipid plaque from which they emerged. Their conclusion is based on the confocal images of junction between the parent lipid stack and the myelin figures under a fixed set of conditions. However, in a series of experiments that we have done with parent lipid stacks of variable thickness ranging from $7 \mu \mathrm{m}$ to $25 \mu \mathrm{m}$, little correlation was found between the thickness of the parent stack and the diameter of myelin figures that protruded from them. Figure 2 shows examples of myelin figures protruded from lipid stack with average thicknesses of 13,17 , and $24 \mu \mathrm{m}$. As can be seen in this figure, the diameter of majority of myelin figures protruded from $17 \mu \mathrm{m}$ thick lipid plaque is larger than the ones protruded from two other lipid plaques.

Examining more than 300 samples, we realized that a myelin figure does not randomly adopt a diameter. We found groups of myelin figures with similar diameter and structure

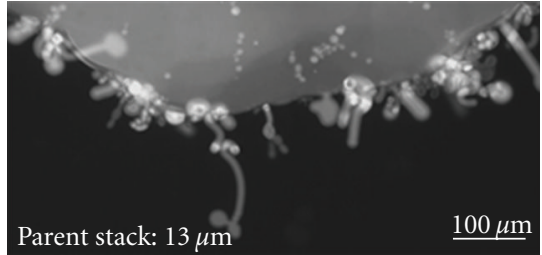

(a)

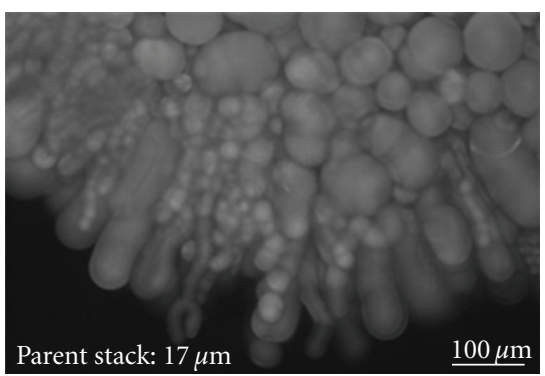

(b)

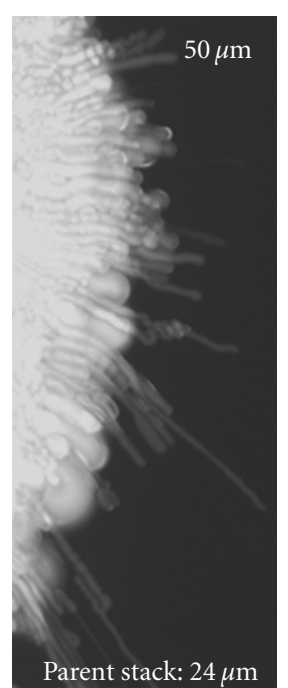

(c)
FIgURE 2: Comparison of average diameter of myelin figures protruded from parent lipid plaques with different thicknesses shows no exact correlation between the thicknesses of parent stack and the diameter of myelin figures.

are growing from separate regions of the parent plaque. All the observed myelin figures can be categorized in three groups: (1) solid tubes, (2) thin tethers, and (3) hollow tubes. Examples of these groups are shown in Figure 3.

As we discussed earlier, myelin figures have the morphology of multilamellar cylindrical tubes. The three observed configurations of solid tubes, thin tethers, and hollow tubes were defined based on two structural terms of multilamellar tubes: (1) core radius and (2) number of lamellae.

Solid tubes represent tubular structures with large number of lamellae. As can be seen in Figure 3(a), in solid tubes, the total diameter of the tube is considerably larger than the diameter of its core. Using fluorescence microscopy, we observed this group of myelin figures as fat shiny tubes with high florescence intensity. Figure 3(a) represents the examples of fluorescent image of solid tubes.

Thin tethers were defined as a group of myelin figures with low number of lamellae and small core radius. The total diameter of thin tethers can be comparable to the diameter of the core. In florescent images, they appeared as shiny thin hair-shaped tubes (Figure 3(b)).

Myelin figures in the last group, hollow tubes, are the ones with low number of lamellae and large core radius. The total thickness of the layers in a hollow tube can be less than the diameter of the core. In fluorescence microscopy, hollow tubes were observed as tubes with very low intensity (Figure 3(c)). These three groups usually could be found in distinct and separated populations in different regions of the parent lipid plaques instead of having mixed population of them from one region. For example, Figure 1(b) represents an example of this fact where solid tubes and hollow ones are formed from different regions of lipid plaque.

The exact origin of the different configuration in different myelin figure groups is not clearly understood. However, 


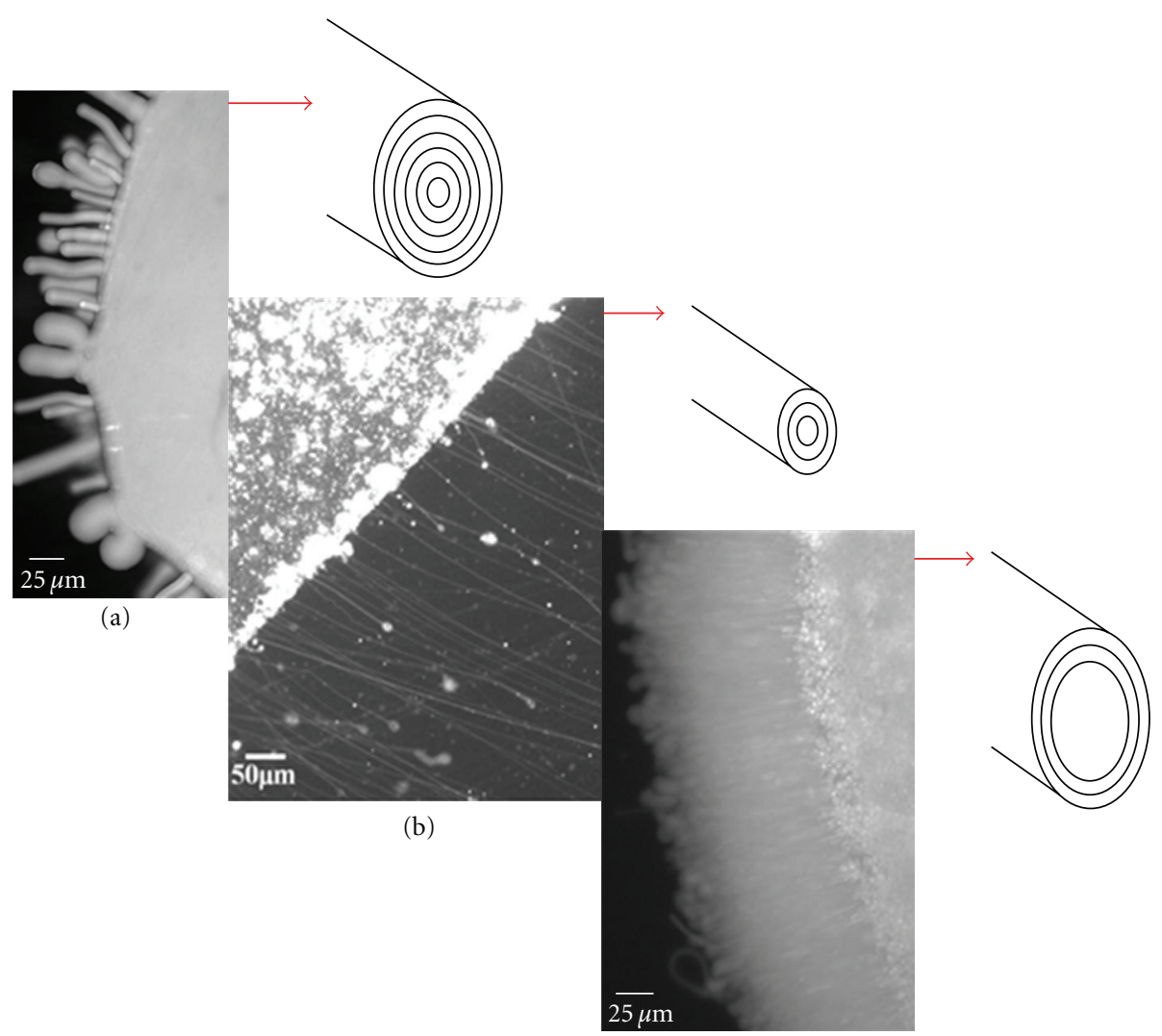

(c)

FIGURE 3: Wide-area fluorescent micrographs illustrating three major types of tubule morphologies observed upon the hydration of dry stack lipid doped with $1 \mathrm{~mol} \%$ Texas Red by water above their phase transition temperature. (a) Solid tubes: large number of lamellar. (b) Thin tethers: low number of lamellae with small core radius. (c) Hollow tubes: low number of lamellae with large core radius.

we suspect that there is a direct relation between the local characteristic of the parent lipid plaque and morphology of the myelin figures. Solid tubes are normal and the most common structure of myelin figures. We observed that the thin tethers can be protruded from the area that the lipid plaque had less wrinkling in the surface. Apparently, wrinkling (as reservoir of lipid) decreases the membrane tension. On the other hand, hollow tubes are constantly observed to be grown from the regions of lipid plaques that are unpinned from the substrate and can have lower membrane tension than normal. Figure 4 shows an example of this fact for a hollow tube in a very reproducible experiment described below. A dry DMPC plaque, which was pinned to the substrate, was immersed under water for couple of minutes at temperature below the phase transition of DMPC $\left(T_{m} \approx 24^{\circ} \mathrm{C}\right)$. Note that myelin figures cannot be formed below $T_{m}$. This causes some parts of the edge of the drop to be unpinned from the substrate but still attached to the whole drop. Now we have two distinct regions of lipid: pinned and unpinned. The temperature increased very slowly to the above phase transition temperature, where the myelin figures can be formed. Interestingly, always, two very distinct populations of myelin figures were observed, hollow and solid tubes (e.g., see Figure 4(a1)). Hollow tubes protrude from the unpinned region and Solid tubes from the pinned region of lipid plaque. By the time, when other parts of the plaque become unpinned, the solid tubes are replaced by hollow tubes. Snapshots of this process are shown in Figure 4, (a1)-(a3). To further validate this hypothesis, we did the similar experiment by letting the whole lipid plaque to be unpinned and suspended from the substrate at temperature lower than $T_{m}$. In this case, when we increase the temperature to above $T_{m}$, only one population of myelin figures grows (Figure 4(b)).

To discriminate between the hollow and solid morphologies of myelin tubes, we relied on the fluorescent emission from the trace concentrations of lipid soluble dyes embedded within the lipid phase. The dramatic differences in the fluorescence intensities from the well-focused images of populations of myelin figures observed in single samples within single wide-field fluorescent micrographs $(500 \mu \mathrm{m} \times 400 \mu \mathrm{m})$ can stem from a variety of sources. While small variations (within 5-10\%) can result from subtle differences in lipid microenvironment, the large variations we observed indicate substantial differences in fluorophore populations. Furthermore, the lipid-conjugated fluorophores used were not sensitive to lipid packing or phase state, thus, allowing us to conclude that their intensity difference does not reflect their preferential accumulation or partitioning into one morphology (e.g., solid versus hollow) 


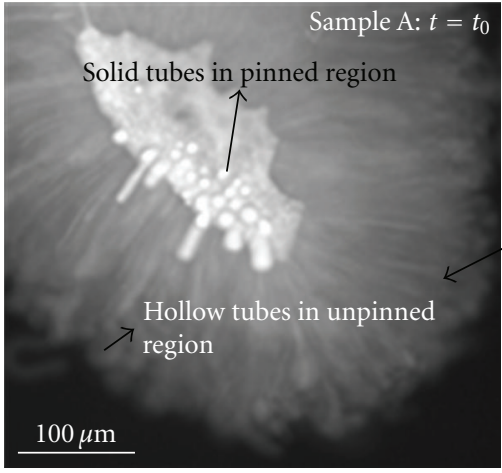

(a1)

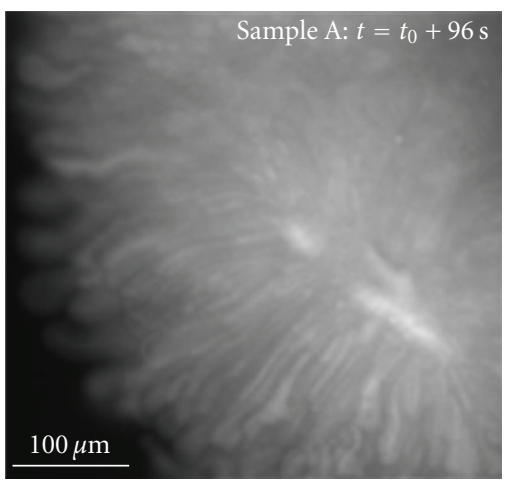

(a3)

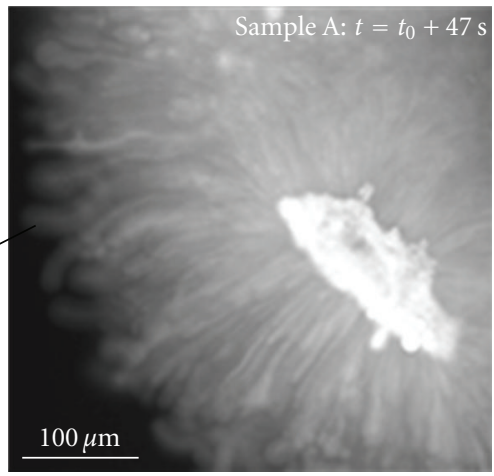

(a2)

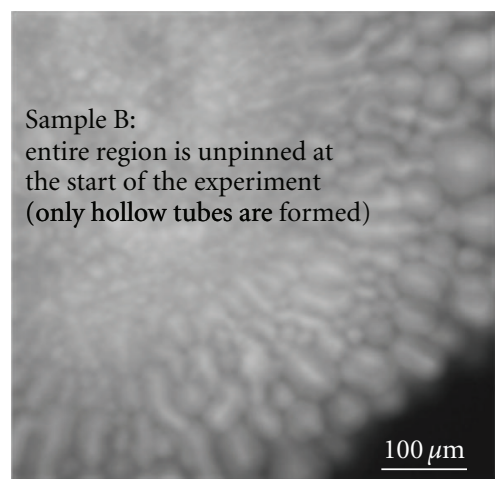

(b)

FIGURE 4: (a1)-(a3) Epifluorescence micrographs reveal coexistence of solid and hollow tubes emanating from single plaque of DMPC (sample A) while edge of the plaque is unpinned from the substrate and its central part is pinned. Protrusion is formed when initially dry lipid plaque is hydrated below $T_{m}$ and then heated to above its $T_{m}$ value. Solid tubes appear from the pinned region and hollow tubes protrude from the unpinned region (a1) and (a2). Over time, the entire plaque becomes unpinned, and all solid tubes retract and are replaced by hollow tubes (a3). (b) Fully suspended DMPC plaques in water produce exclusively hollow tubes. (DMPC is doped with 0.75 mol\% TR.)

over the other. Based on these considerations, we assumed direct proportionality between fluorescence intensity and the average lipid density. Following this criterion, myelin figures with faint fluorescence (in well-focused image) can be conveniently categorized as "hollow" tubes whereas ones with strong fluorescence (fourfold above the hollow ones) can be assigned to the "solid" tube. (Note that to have clear images of hollow or solid tubes at a same spot, we usually need to change the focus).

Hollow tubes can be best visualized by differentially staining the lipid and the aqueous phase. To illustrate characterization of hollow tubes, below we performed a dual probe experiment. Here, a plaque of dry POPC, doped with $1 \mathrm{~mol} \%$ TR-DHPE, was hydrated using $5 \mathrm{~mL}$ solution of fluorescently doped water $\left(5 \times 10^{-4} \mathrm{mg} / \mathrm{mL}\right.$ Fluorescein/water). The plaque was pinned to the underlying substrate. After formation of myelin figures, the fluoresceindoped water in the aqueous phase was gradually replenished by undoped DI water (by replacing $1 \mathrm{~mL}$ solution by DI water in multiple (5-10) iterations). Figure 5 displays a twocolor fluorescent image (a) as well as single color images (b) and (c). The images were obtained when part of the parent plaque had unpinned from the substrate, thus, providing condition for the formation of hollow tubes. Unfocused hollow myelin figures often appeared as faint and blurry features surrounding the parent plaque. However, wellfocused ones (such as seen in Figure 5) revealed distinct fluorescence from both the lipid and the aqueous phase, consistent with the core-shell structure of hollow myelin figures discussed in Figure 3(c).

\section{Conclusion}

Employing epiflouerescence microscopy, we studied the lifetime and configuration of myelin figures in experiment of lipid hydration. There were direct correlations between the lifetime of myelin figures and the experimental conditions such as temperature and density of the parent stack and the hydrophobicity of the substrate. Denser parent stack on hydrophobic substrate at low temperature can produce more stable myelin figures with greater lifetime (more than 13 hours). There was evidence that indicated the diameter of myelin figures was related to the local characteristics of the parent stack. However, such characteristics were not easily quantifiable in the experiments. Specifically, we observed that distinct populations of myelin figures with similar configuration protrude from distinct regions of parent stack. Evidenced by fluorescent images, we classified 


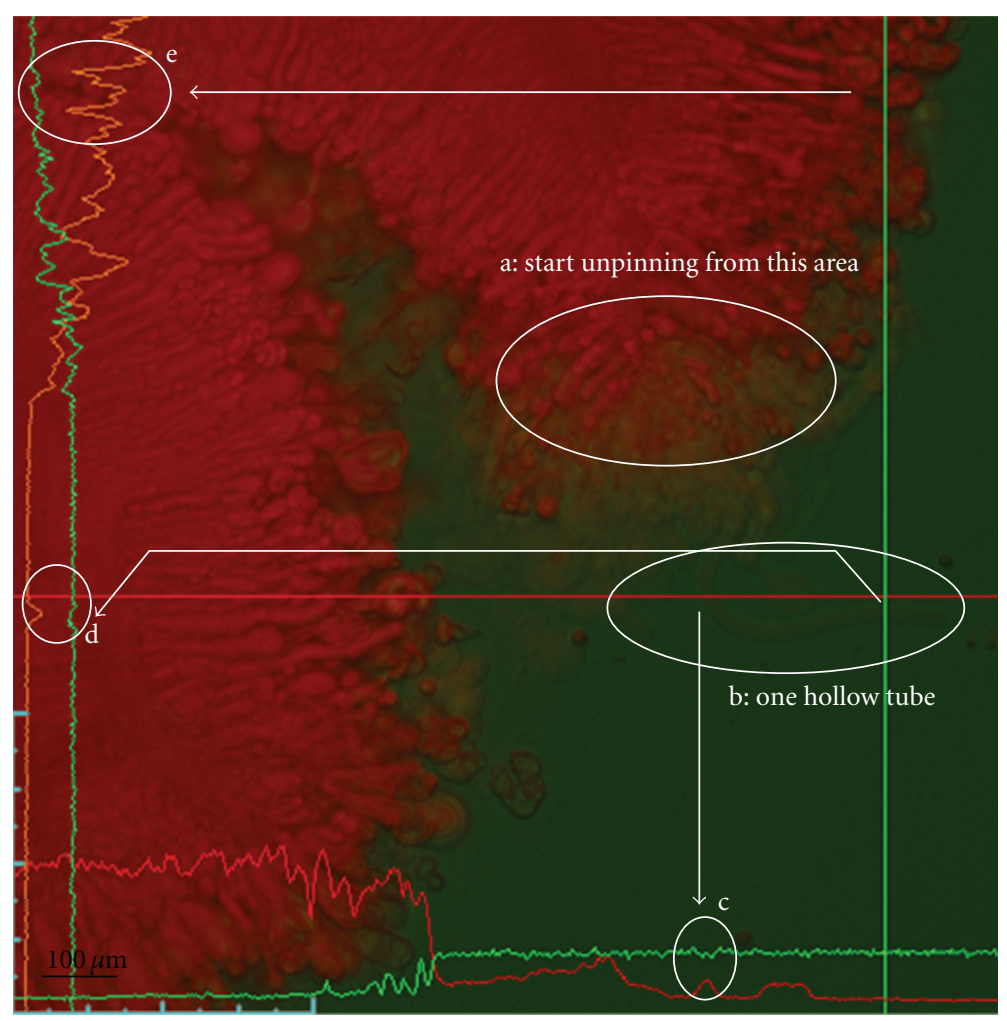

(a)

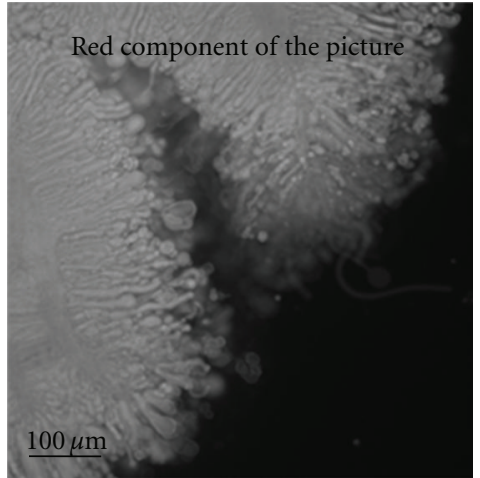

(b)

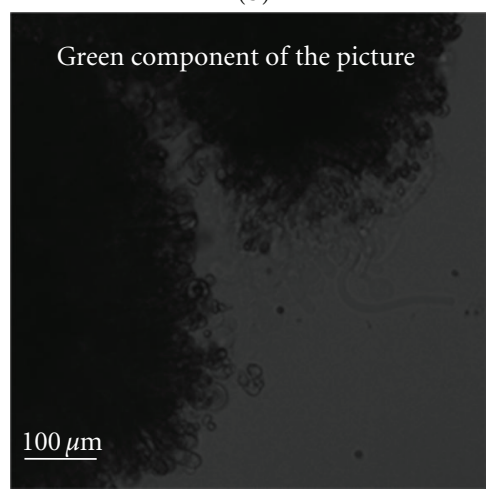

(c)

FIGURE 5: POPC, $1 \mathrm{~mol} \%$ TR-DHPE, room temperature, hydrate solution of fluorescein/DI water $5 \times 10^{-4} \mathrm{mg} / \mathrm{mL}$ Fluorescein. (a) Shows the two-color image; (b) and (c) are the same image with only one-color component (red (lipid) or green (water)). Region a: in (a) shows the area where the plaque of lipid start unpinning from the substrate. So, the myelin figures growing from that area have a hollow shape. Region b: shows one of these hollow tubes. (Circles $\mathrm{c}$ and d) refer to the intensity of two areas in the hollow tube which can be compared with the environment of a solid tube (Circle e).

all the observed myelin figures in three major groups of (1) solid tubes, (2) thin tethers, and (3) hollow tubes which were analyzed in this paper. We defined first group as multilamellar tubes with a large number of lamellae in which the total thickness of multilamellar layer is much thicker than the core radius. The second and third groups consist of myelin figures with a low number of lamellae. In the second group, the total thickness of layers is comparable to the core diameter. However, in the third group, the core diameter is very large compared to the total thickness of layers in a way that the myelin figures look like hollow tubes. Solid tubes are the most common structure of myelin figures. On the basis of our observation, thin tethers are more prone to be formed from the source lipid with higher membrane tension. On the other hand, our experiments showed that hollow tubes are mostly formed when part of the parent lipid plaque is unpinned from substrate so its tension was decreased. In this occasion, an abrupt change from solid tube to hollow ones can be observable reproducibly.

\section{References}

[1] L. N. Zou and S. R. Nagel, "Stability and growth of single myelin figures," Physical Review Letters, vol. 96, no. 13, Article ID 138301, pp. 1-4, 2006.
[2] W. Harbich and W. Helfrich, "The swelling of egg lecithin in water," Chemistry and Physics of Lipids, vol. 36, no. 1, pp. 3963, 1984.

[3] W. Stoeckenius, "OsO4-Fixierung intrazellulärer myelinfiguren,” Experimental Cell Research, vol. 13, no. 2, pp. 410-414, 1957.

[4] S. C. Watkins and R. D. Salter, "Functional connectivity between immune cells mediated by tunneling nanotubules," Immunity, vol. 23, no. 3, pp. 309-318, 2005.

[5] D. G. Dervichian, "Swelling and molecular organisation in colloidal electrolytes," Transactions of the Faraday Society, vol. 42, pp. B180-B187, 1946.

[6] M. Besisi, Living Blood Cells and Their Ultrastructure, Springer, Berlin, Germany, 1973.

[7] N. Basic-Jukic, M. Coric, P. Kes, L. J. Bubic-Filipi, J. Pasini, and I. Mokos, "Anderson-Fabry disease in kidneys from deceased donor," American Journal of Transplantation, vol. 7, no. 12, pp. 2829-2833, 2007.

[8] F. R. Poulain, L. Allen, M. C. Williams, R. L. Hamilton, and S. Hawgood, "Effects of surfactant apolipoproteins on liposome structure: implications for tubular myelin formation," American Journal of Physiology, vol. 262, no. 6, pp. L730-L739, 1992.

[9] H. Fehrenbach, S. Tews, A. Fehrenbach et al., "Improved lung preservation relates to an increase in tubular myelinassociated surfactant protein A," Respiratory Research, vol. 6, article 60, 2005. 
[10] R. J. Sanderson and A. E. Vatter, "A mode of formation of tubular myelin from lamellar bodies in the lung," Journal of Cell Biology, vol. 74, no. 3, pp. 1027-1031, 1977.

[11] A. Policard, A. Collet, and S. Pregermain, "Electron microscopic study of the first stages of lipophanerosis in histiocytes," Comptes Rendus Hebdomadaires Des Seances De L Academie Des Sciences, vol. 246, no. 25, pp. 3405-3406, 1958.

[12] J. R. Huang, L. N. Zou, and T. A. Witten, "Confined multilamellae prefer cylindrical morphology: AAAA theory of myelin formation," European Physical Journal E, vol. 18, no. 3, pp. 279-285, 2005.

[13] I. Sakurai, T. Suzuki, and S. Sakurai, "Cross-sectional view of myelin figures," Biochimica Et Biophysica Acta, vol. 985, no. 1, pp. 101-105, 1989.

[14] M. Buchanan, J. Arrault, and M. E. Cates, "Swelling and dissolution of lamellar phases: role of bilayer organization," Langmuir, vol. 14, no. 26, pp. 7371-7377, 1998.

[15] A. P. Kennedy, J. Sutcliffe, and J. X. Cheng, "Molecular composition and orientation in myelin figures characterized by coherent anti-stokes Raman scattering microscopy," Langmuir, vol. 21, no. 14, pp. 6478-6486, 2005.

[16] W. J. Benton, K. H. Raney, and C. A. Miller, "Enhanced videomicroscopy of phase transitions and diffusional phenomena in oil-water-nonionic surfactant systems," Journal of Colloid And Interface Science, vol. 110, no. 2, pp. 363-388, 1986.

[17] L. Reissig, D. J. Fairhurst, J. Leng, M. E. Cates, A. R. Mount, and S. U. Egelhaaf, "Three-dimensional structure and growth of myelins," Langmuir, vol. 26, no. 19, pp. 15192-15199, 2010.

[18] E. Evans, H. Bowman, A. Leung, D. Needham, and D. Tirrell, "Biomembrane templates for nanoscale conduits and networks," Science, vol. 273, no. 5277, pp. 933-935, 1996.

[19] T. Roopa, N. Kumar, S. Bhattacharya, and G. V. Shivashankar, "Dynamics of membrane nanotubulation and DNA selfassembly," Biophysical Journal, vol. 87, no. 2, pp. 974-979, 2004.

[20] L. N. Zou, "Myelin figures: the buckling and flow of wet soap," Physical Review E, vol. 79, no. 6, Article ID 061502, 2009.

[21] C. D. Santangelo and P. Pincus, "Coiling instabilities of multilamellar tubes," Physical Review E, vol. 66, no. 6, Article ID 061501, 2002.

[22] K. C. Lin, R. M. Weis, and H. M. McConnell, "Induction of helical liposomes by $\mathrm{Ca}^{2+}$-mediated intermembrane binding," Nature, vol. 296, no. 5853, pp. 164-165, 1982.

[23] V. Frette, I. Tsafrir, M. A. Guedeau-Boudeville, L. Jullien, D. Kandel, and J. Stavans, "Coiling of cylindrical membrane stacks with anchored polymers," Physical Review Letters, vol. 83, no. 12, pp. 2465-2468, 1999.

[24] I. Derényi, F. Jülicher, and J. Prost, "Formation and interaction of membrane tubes," Physical Review Letters, vol. 88, no. 23, pp. 2381011-2381014, 2002. 


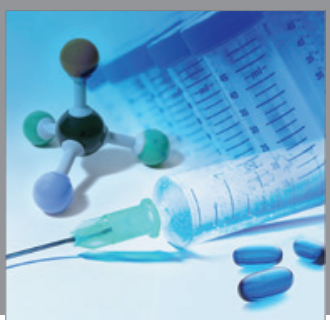

International Journal of

Medicinal Chemistry

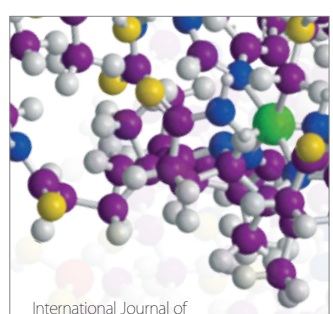

Carbohydrate Chemistry

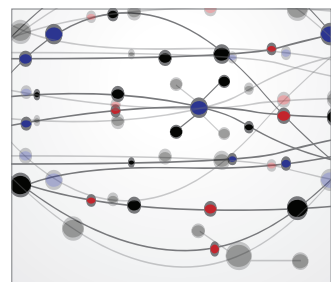

The Scientific World Journal
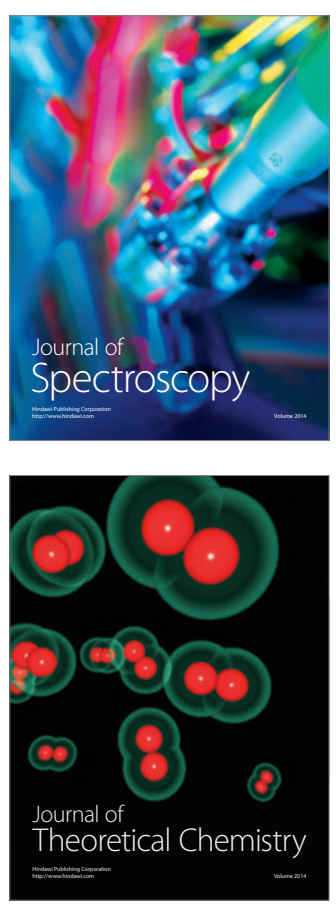
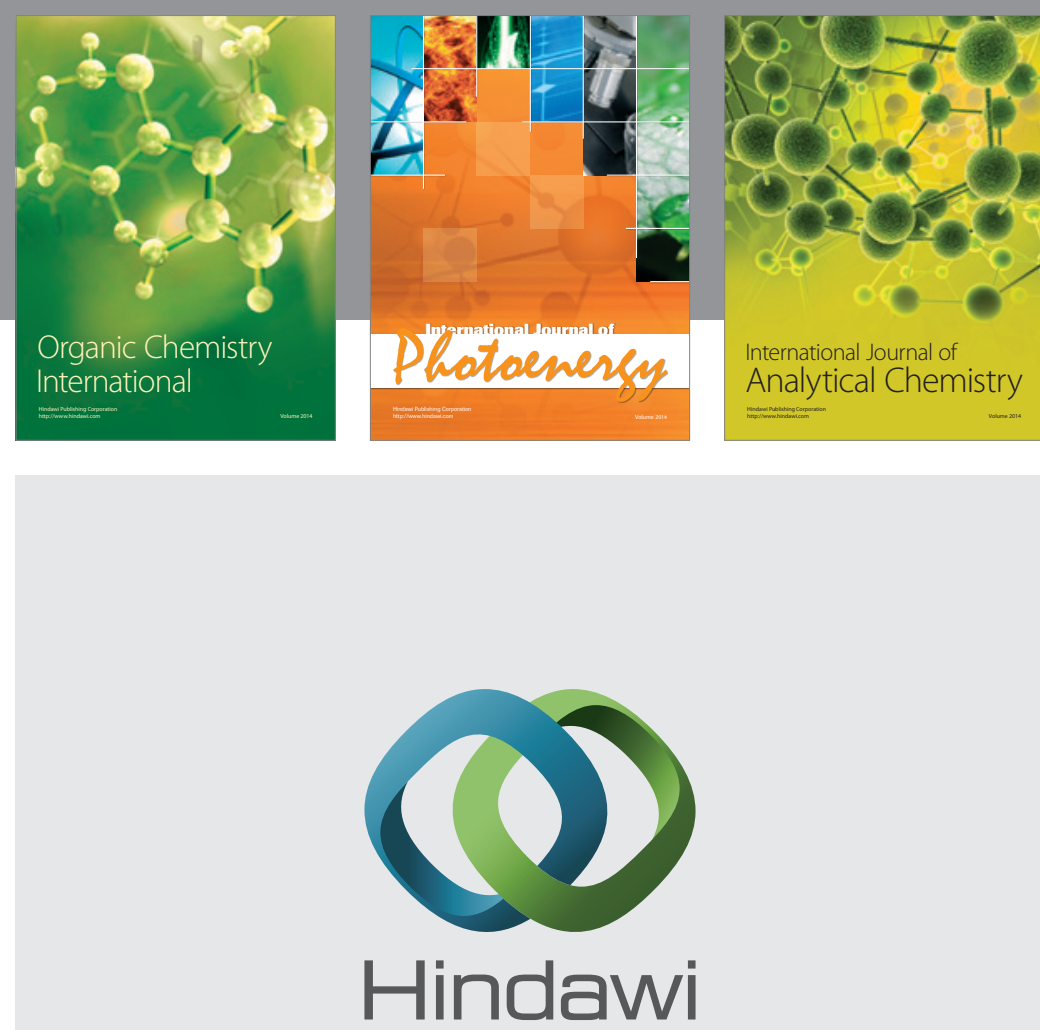

Submit your manuscripts at

http://www.hindawi.com
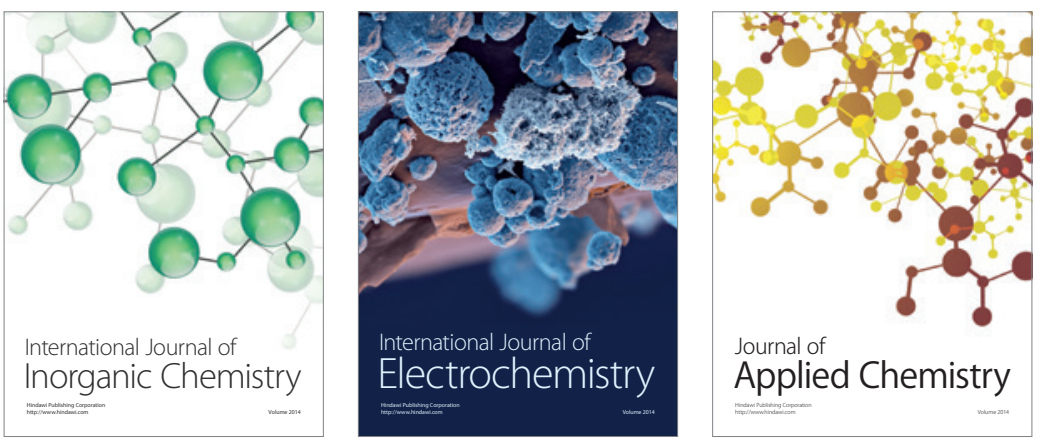

Journal of

Applied Chemistry
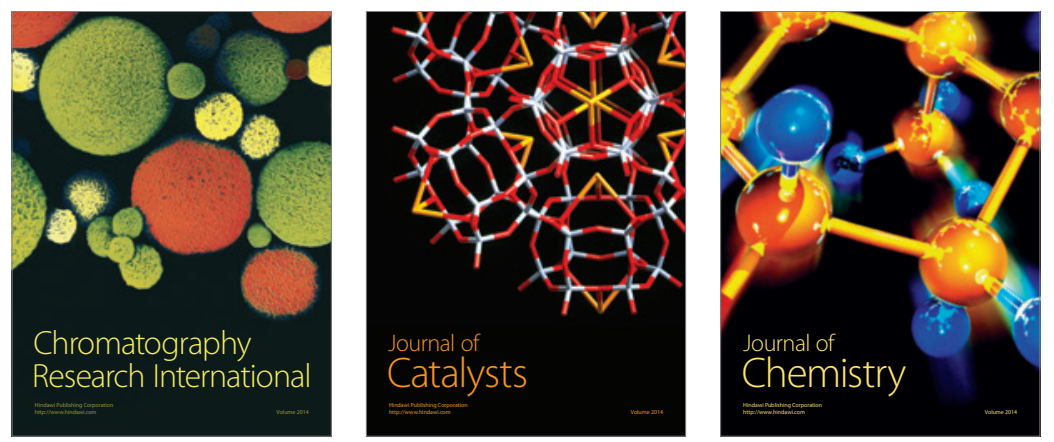
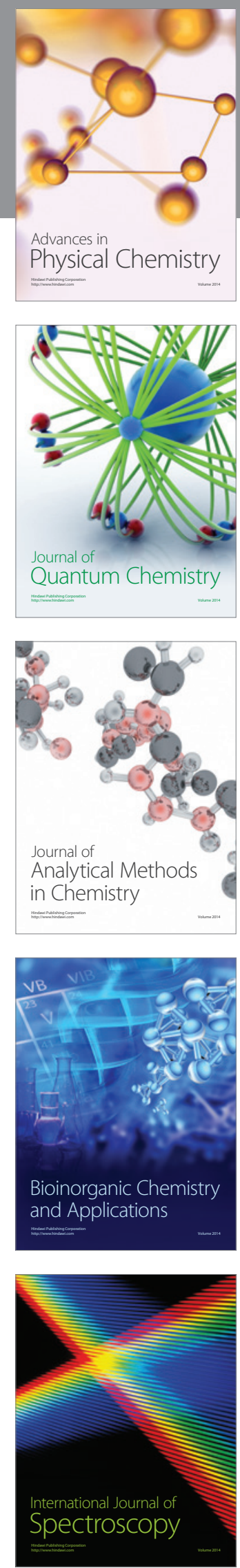